\title{
Theoretical and Computational Studies on Compression Process of Gas with Water Droplets
}

\author{
Palani Kumar Chidambaram, Abhay Mohan², Abhilash Suryan³, Heuy Dong Kim² \\ ${ }^{1}$ FMTRC, Daejoo Machinery Co Ltd \\ 1028, Woram-Dong, Dalseo-Gu, Daegu, Korea. \\ kumar@djair.co.kr \\ ${ }^{2}$ Dept of Mechanical Engineering, Andong National University \\ Andong, Korea \\ mohan@ anuis.andong.ac.kr; kimhd@andong.kr \\ ${ }^{3}$ Department of Mechanical Engineering, College of Engineering Trivandrum, \\ Thiruvananthapuram, Kerela, India. \\ suryan@cet.ac.in
}

\section{Extended Abstract}

In a gas turbine, the compressor typically consumes a major portion of the power produced by the turbine. Therefore, a reduction in the compressor work significantly improves the gas turbine power output. The compressor work increases monotonically with the air temperature. Inlet fogging is a well known technology used to cool the air before it enters the compressor. Fine mist is sprayed into the air ahead of the compressor. The mist evaporates completely before the compressor reducing the air temperature. A lower initial temperature results in a lower compression work. Another interesting idea is to use the large latent heat of vaporization of water for cooling the air inside compressor; a technique called wet compression. The evaporating water droplets absorb the heat as the air is being compressed. This heat removal reduces the air temperature progressively during the compression process and thus effectively reduces the compressor work. Although the use of water droplets to cool the air entering compressor had been studied decades ago both theoretically, numerically and experimentally, there is a renewed interest in this research, recently within the past two decades $[1,2]$.

In the present study, the two phase compression of air - water droplet mixture is numerically and analytically studied and the results are compared and contrasted. The work is focused on understanding the fundamental concepts associated with two phase compression. Hence, a simple piston-cylinder system is used as the simulation domain for the compression of air - water droplet mixture. Axi-symmetric numerical computations were performed using a commercial CFD solver by solving URANS equations with water droplets being modelled by Discrete Phase Method. Analytical predictions were made by iteratively solving thermodynamic relations of compression process coupled with heat and mass transfer relations between air and water droplets. During both numerical and analytical computations, thermodynamic properties such as temperature, pressure, enthalpy, entropy and relative humidity inside the cylinder are monitored and investigated. Parametric studies are then performed to identify the effects of initial relative humidity of air, overspray percentage of water droplets, droplet diameter, and compression rate of the piston individually.

Initial results from the study indicate that the reduction in compressor work is higher for larger overspray percentage of water droplets, droplets of smaller diameter, and slower compression rate. The initial relative humidity of air however is seen to be insignificant in the compressor work reduction. While the results from both numerical and analytical predictions agree qualitatively with each other, they differ quantitatively. The major reason for deviation between the computations is attributed to the presence of spatial gradients in the numerical solver.

\section{References}

[1] Q. Zheng, Y. Sun, S. Li, and Y. Wang, "Thermodynamic Analyses of Wet Compression Process in the Compressor of Gas Turbine," Journal of Turbomachinery, vol. 125, pp. 489-496, 2003. 
[2] A. J. White and A. J. Meacock, "An Evaluation of the Effects of Water Injection on Compressor Performance," ASME Journal of Engineering for Gas Turbine and Power, vol. 126, no. 4, pp. 748-754, 2004. 